\title{
Low versus high pulse oxygen saturation directed oxygen therapy in critically ill patients: a randomized controlled pilot study
}

\author{
Xiaobo Yang ${ }^{1,2}$, You Shang ${ }^{1,2}$, Shiying Yuan ${ }^{1,2}$ \\ ${ }^{1}$ Department of Critical Care Medicine, ${ }^{2}$ Institute of Anesthesiology and Critical Care Medicine, Union Hospital, Tongji Medical College, Huazhong \\ University of Science and Technology, Wuhan 430022, China \\ Contributions: (I) Conception and design: X Yang, Y Shang; (II) Administrative support: S Yuan; (III) Provision of study material or patients: All \\ authors; (IV) Collection and assembly of data: All authors; (V) Data analysis and interpretation: X Yang, S Yuan; (VI) Manuscript writing: All authors; \\ (VII) Final approval of manuscript: All authors. \\ Correspondence to: Shiying Yuan. Department of Critical Care Medicine, Union Hospital, Tongji Medical College, Huazhong University of Science \\ and Technology, Wuhan 430022, China. Email: shiying_yuan@163.com.
}

\begin{abstract}
Background: Data on the safety and feasibility of pulse oxygen saturation $\left(\mathrm{SpO}_{2}\right)$ directed oxygen therapy in mainland China are scarce. The aim of this pilot study was to test the feasibility of $\mathrm{SpO}_{2}$ directed oxygen therapy and to calculate sample size base on differences in 28-day mortality rates for a large sample-sized randomized trial.
\end{abstract}

Methods: This prospective pilot study enrolled 214 adult patients with an expected intensive care unit (ICU) stay of more than 72 hours. Patients were randomized into a low $\mathrm{SpO}_{2}$ group $\left(\mathrm{SpO}_{2} 90-95 \%\right)$ or high $\mathrm{SpO}_{2}$ group ( $\mathrm{SpO}_{2}$ 96-100\%). The primary outcome was 28-day mortality.

Results: One hundred patients were included in the low $\mathrm{SpO}_{2}$ group, and 114 patients were included in the high $\mathrm{SpO}_{2}$ group. The demographic and baseline characteristics were not different. The time-weighted $\mathrm{SpO}_{2}$ average was significantly lower in the low $\mathrm{SpO}_{2}$ group than in the high $\mathrm{SpO}_{2}$ group [mean \pm standard deviation (SD), $95.7 \% \pm 2.3 \%$ vs. $98.2 \% \pm 1.8 \%, \mathrm{P}<0.001]$. Twenty-six patients $(26 \%)$ in the low $\mathrm{SpO}_{2}$ group died within 28 days after inclusion, while 37 patients $(32.5 \%)$ in the high $\mathrm{SpO}_{2}$ group died $(\mathrm{P}=0.301)$. The time to death within 28 days between the two groups was not different $(\mathrm{P}=0.284)$.

Conclusions: $\mathrm{SpO}_{2}$ directed oxygen therapy in critically ill patients was feasible. Our pilot trial necessitates and rationalizes our large-sample multicenter trial.

Keywords: oxygen therapy; pulse oxygen saturation $\left(\mathrm{SpO}_{2}\right)$; mortality

Submitted Apr 12, 2019. Accepted for publication Sep 23, 2019.

doi: $10.21037 /$ jtd.2019.09.66

View this article at: http://dx.doi.org/10.21037/jtd.2019.09.66

\section{Introduction}

Oxygen therapy is commonly used. The speculation of increased oxygen demand and decreased oxygen supply in critically ill patients promotes the use of oxygen therapy in intensive care units (ICUs). However, hyperoxia can be harmful in critically ill patients with specific diseases. Examples are myocardial infarction (MI) patients and patients resuscitated to the return of spontaneous circulation after cardiac arrest. In the Air Versus Oxygen in Myocardial Infarction (AVOID) trial, Stub and colleagues (1) found that hyperoxia caused more cardiac injury in patients with STelevation MI but without hypoxia. Kilgannon and colleagues $(2,3)$ found that arterial hyperoxia following resuscitation from cardiac arrest was associated with increased in-hospital mortality, and a pulse oxygen saturation $\left(\mathrm{SpO}_{2}\right)$ of $94-98 \%$ was recommended (4).

In general, for critically ill patients without severe acute respiratory syndrome (ARDS), evidence regarding oxygen therapy is scarce. Recently, Panwar and colleagues (5) found that compared to a liberal oxygen strategy $\left(\mathrm{SpO}_{2} \geq 96 \%\right.$ ), a conservative oxygen strategy $\left(\mathrm{SpO}_{2}\right.$ of $\left.88-92 \%\right)$ was feasible 
in patients receiving invasive mechanical ventilation. Girardis and colleagues (6) found that control oxygen therapy (target $\mathrm{SpO}_{2}$ of $94-98 \%$ or $\mathrm{PaO}_{2}$ of $70-100 \mathrm{mmHg}$ ) resulted in lower ICU mortality than conventional oxygen therapy $\left(\mathrm{SpO}_{2} \geq 97 \%\right)$ in the randomized clinical trial called Oxygen-ICU. However, the Oxygen-ICU trial was a singlecentered study with only 480 included patients that was terminated early after an unplanned interim analysis $(6,7)$.

We hypothesized that low $\mathrm{SpO}_{2}$-targeted oxygen therapy was safe and would reduce the 28-day mortality in patients staying longer than 72 hours in ICUs. We assumed a 28-day mortality rate of $30 \%$ in the high $\mathrm{SpO}_{2}$ group based on our retrospective data and estimated that the enrollment of 2,148 patients would enable the detection of an absolute betweengroup difference of $6 \%$ in 28-day mortality at a two-sided significance level of 0.05 with a power of $90 \%$ and a dropout rate of $20 \%$. We conducted a single-center randomized controlled pilot study of $10 \%$ of the estimated sample size in our ICU to test the feasibility of $\mathrm{SpO}_{2}$ directed oxygen therapy and to rationalize our large-sample multicenter trial.

\section{Methods}

\section{Study design and patient screening}

The protocol had been previously approved by the ethics committee of Tongii Medical College, Huazhong University of Science and Technology, with which our hospital is affiliated. Written informed consent was obtained from each patient's legal surrogate.

All patients aged 18 years or older and admitted to the ICU with an expected length of stay of 72 hours or longer were screened. Estimation of ICU stay was made by one of the three authors, who has been working in a general ICU for at least of 7 years. The exclusion criteria included the following: ICU readmission, patients with acute exacerbation of chronic obstruction disease, patients with severe acute respiratory distress syndrome [defined as $\mathrm{PaO}_{2}$ /fraction of inspiratory oxygen $\left(\mathrm{FiO}_{2}\right) \geq 100 \mathrm{mmHg}$, inclusion in another interventional trial, a decision to withhold life-sustaining treatment, pregnancy, paraquat poisoning and not having been screened within 12 hours after admission.

\section{Randomization and study treatment}

On inclusion, patients were randomized by a computerized random-number generator into a low $\mathrm{SpO}_{2}$ group or a high $\mathrm{SpO}_{2}$ group. The randomization sequence was concealed in sequentially numbered, closed, opaque envelopes that were opened after patient study inclusion. In the low $\mathrm{SpO}_{2}$ group, the $\mathrm{SpO}_{2}$ target was $90-95 \%$, with the $\mathrm{FiO}_{2}$ as low as possible. In the high $\mathrm{SpO}_{2}$ group, the $\mathrm{SpO}_{2}$ target was 96-100\%, with $\mathrm{FiO}_{2}$ no lower than $30 \%$. If $\mathrm{SpO}_{2}$ was unattainable, $\mathrm{SO}_{2}$ from arterial blood gas was used. During intubation, airway suction, tracheostomy and other critical situations such as hemodynamic collapse, physicians could temporarily elevate $\mathrm{FiO}_{2}$ to $100 \%$. If severe adverse events that were possibly associated with hypoxia occurred in the low $\mathrm{SpO}_{2}$ group, the treating physicians could withdraw the patient. All other actions, including (but not limited to) noninvasive ventilation, intubation, ventilator setting, vasoactive drug support, radiological examination, arterial blood gas sampling and microbiological sampling, were performed according to institutional protocols. The treating team was aware of the $\mathrm{SpO}_{2}$ target and adjusted the $\mathrm{FiO}_{2}$ accordingly, but the included patients were unaware of their treatment group.

\section{Data collection}

Demographic data, Acute physiology, Age, and Chronic Health Evaluation II (APACHE II) scores, type of admission (medical or surgical), and Charlson comorbidity scores were collected. $\mathrm{FiO}_{2}$ and $\mathrm{SpO}_{2}$ were recorded every six hours until 14 days, death or ICU discharge, whichever came first. The time-weighted averages of $\mathrm{FiO}_{2}$ and $\mathrm{SpO}_{2}$ were calculated as the mean value of 2 consecutive measurements multiplied by the time (hours) between the measurements and divided by all the time combined (hours). The timeweighted average $\mathrm{pO}_{2}$ from the arterial blood gas analysis was calculated in the same manner. The use of invasive mechanical ventilation and renal replacement therapy were recorded daily. Follow-up phone calls were made every week after discharge until 28 days after inclusion.

\section{Outcomes}

The primary outcome was 28-day mortality. The secondary outcome was ICU mortality. It is common that in China, patients' legal surrogates do not want terminal patients to die in the hospital, and they would check out the patients in the agonal stage. The patients who were checked out in the agonal stage and actually died within 24 hours were considered to have died in our ICU. 


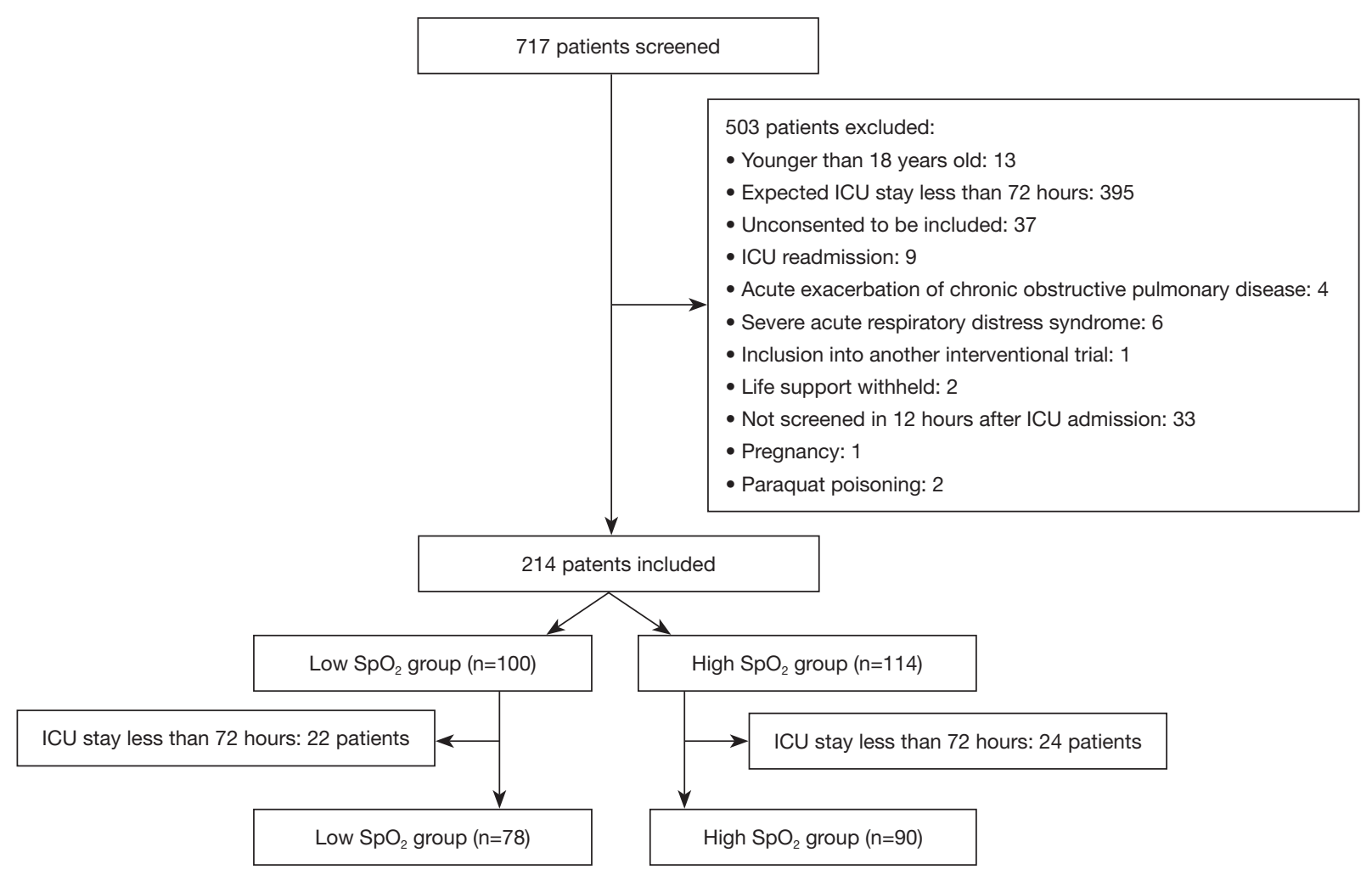

Figure 1 Screening process and patients inclusion. ICU, intensive care unit; $\mathrm{SpO}_{2}$, pulse oxygen saturation.

\section{Statistical analysis}

For all randomized patients, an intent-to-treat (ITT) analysis was used. A 72-hour subgroup analysis was also used for randomized patients who stayed in the ICU longer than 72 hours. Continuous data were expressed as the mean \pm SD or median interquartile range (IQR). For each continuous variable, normality was determined by two-way analysis of variance. If the $\mathrm{F}$ value was statistically significant, an unpaired two-tailed Student's $t$-test was used to evaluate differences between groups. Otherwise, the Mann-Whitney U test was used. Categorical data were expressed as counts (percentage in \%) and analyzed by the chi-square test. The effect of oxygen therapy on the time to death was assessed using Kaplan-Meier analysis and the log-rank test. A P value of $<0.05$ was regarded as statistically significant. We used Stata/IC 15.1 software (StataCorp, Texas, USA) for the statistical analysis.

\section{Results}

\section{Study population}

From February 17th to October 24th in 2017, we screened
717 patients and included 214 patients (Figure 1). For the ITT analysis, data from 100 patients in the low $\mathrm{SpO}_{2}$ group and 114 patients in the high $\mathrm{SpO}_{2}$ group were used. For the 72-hour subgroup analysis, data from 78 patients in the low $\mathrm{SpO}_{2}$ group and 90 patients in the high $\mathrm{SpO}_{2}$ group were used. The median age, gender, APACHE II score, type of admission, Charlson comorbidity score, mechanical ventilation and renal replacement were similar between the two groups (Table 1). More respiratory failure patients were included in the low $\mathrm{SpO}_{2}$ group than in the high $\mathrm{SpO}_{2}$ group ( $63 \%$ vs. $46.5 \%, \mathrm{P}=0.016)$.

\section{Oxygen control and outcomes}

In the ITT population, the time-weighted $\mathrm{SpO}_{2}$ average during the ICU stay was significantly lower in the low $\mathrm{SpO}_{2}$ group than in the high $\mathrm{SpO}_{2}$ group (mean $\pm \mathrm{SD}$ : $95.7 \% \pm 2.3 \%$ vs. $98.2 \% \pm 1.8 \%, \mathrm{P}<0.001)$ and the timeweighted $\mathrm{FiO}_{2}$ average was significantly lower in the low $\mathrm{SpO}_{2}$ group than in the high $\mathrm{SpO}_{2}$ group median IQR: 33 [25-42] vs. 42 [36-50], $\mathrm{P}<0.001\}$ (Table 2). The timeweighted $\mathrm{pO}_{2}$ averages were borderline significantly 
Table 1 Characteristics of the patients at study inclusion between the two groups

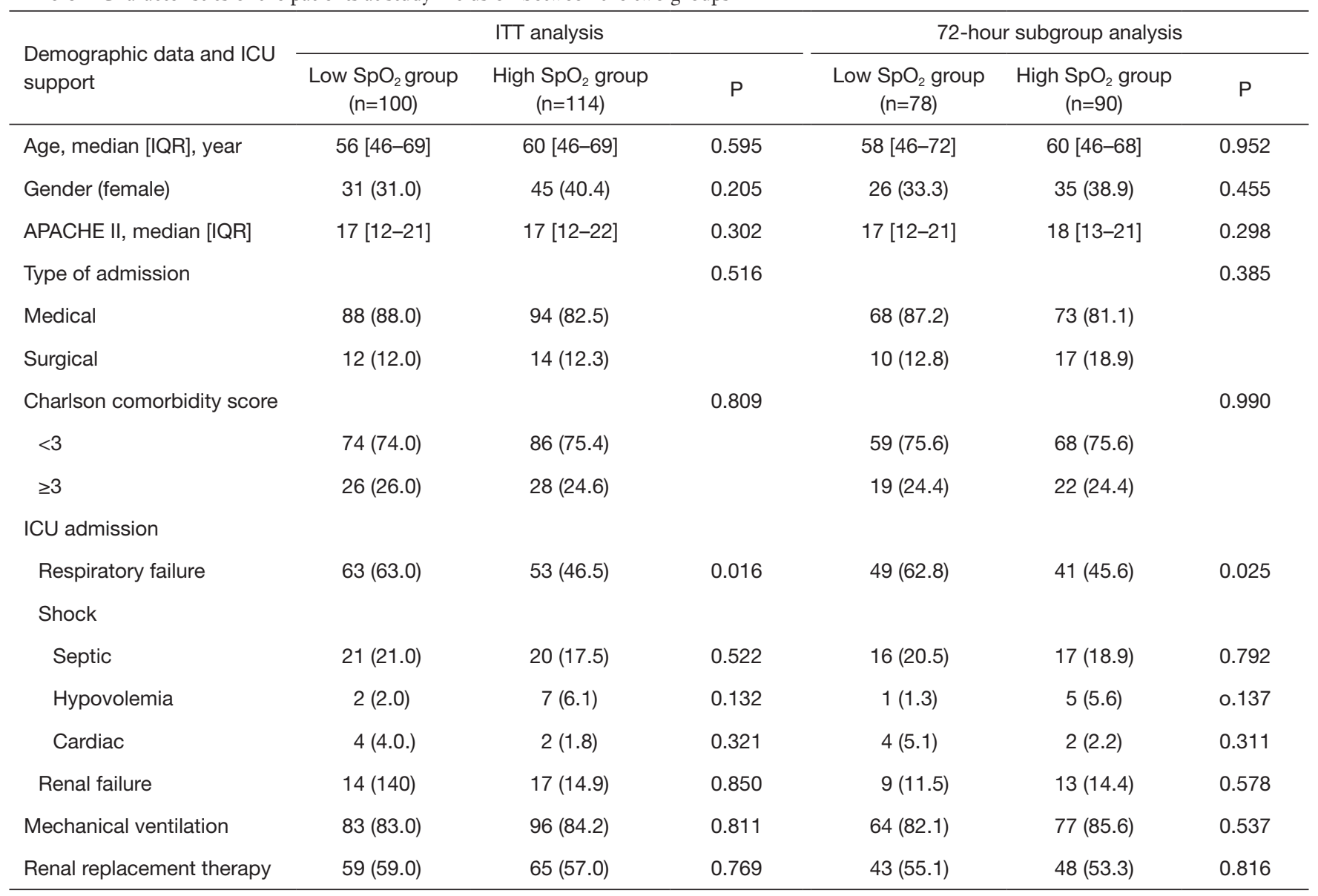

All data were expressed count (percentage in \%) except age and APACHE II. ICU, intensive care unit; ITT, intention to treat; IQR, interquartile range; APACHE II, Acute physiology, Age, and Chronic Health Evaluation II.

Table 2 Oxygen parameters, primary outcome and secondary outcome

\begin{tabular}{|c|c|c|c|c|c|c|}
\hline $\begin{array}{l}\text { Oxygen parameters and } \\
\text { mortality }\end{array}$ & \multicolumn{3}{|c|}{ ITT analysis } & \multicolumn{3}{|c|}{ 72-hour subgroup analysis } \\
\hline $\mathrm{pO}_{2}$, median $[\mathrm{IQR}], \mathrm{mmH}_{\mathrm{g}}$ & 84 [71-99] & $98[79-116]$ & 0.060 & $86[71-101]$ & $98[79-116]$ & 0.108 \\
\hline ICU mortality & $21(21.0)$ & $32(28.1)$ & 0.232 & $11(14.1)$ & $21(23.3)$ & 0.129 \\
\hline
\end{tabular}

Mortality data were expressed count (percentage in \%). ICU, intensive care unit; ITT, intention to treat; SD, standard deviation; IQR, interquartile range; ICU, intensive care unit. 


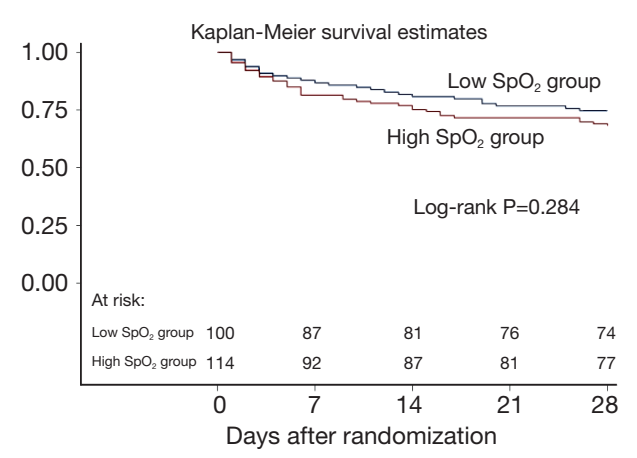

Figure 2 Probability of survival from study randomization through day 28 for patients in the two groups. $\mathrm{SpO}_{2}$, pulse oxygen saturation.

different [median IQR: 84 [71-99] in the low $\mathrm{SpO}_{2}$ group vs. 98 [79-116] in the high $\mathrm{SpO}_{2}$ group, $\mathrm{P}=0.060$ ] (Table 2). The analysis of the 72-hour subgroup population yielded results similar to those of the ITT analysis.

In the ITT population, 26 patients in the low $\mathrm{SpO}_{2}$ group (26\%) died within 28 days after inclusion compared with 37 patients who died in the high $\mathrm{SpO}_{2}$ group $(32.5 \%)$ $(\mathrm{P}=0.301)$ (Table 2). The ICU mortality was not significantly different either $\left(21 \%\right.$ in the low $\mathrm{SpO}_{2}$ group vs. $28.1 \%$ in the high $\mathrm{SpO}_{2}$ group, $\mathrm{P}=0.232$ ) (Table 2). The analysis of the 72-hour subgroup population yielded results similar to those of the ITT analysis with respect to both the primary and secondary outcomes. The time to death within 28 days between the two groups was not different $(\mathrm{P}=0.284)$ (Figure 2).

\section{Discussion}

In our single-center pilot trial, $\mathrm{SpO}_{2}$ directed oxygen therapy in critical patients was feasible. No significant difference in 28-day mortality or in ICU mortality was found between the low $\mathrm{SpO}_{2}$ group and the high $\mathrm{SpO}_{2}$ group.

Although there is considerable evidence regarding oxygen therapy in cardiac ICU patients, evidence pertaining to oxygen therapy in general ICU patients without ARDS is lacking. Stub and colleagues (1) found that hyperoxia caused more cardiac injury, but they declared that the AVOID trial was not powered for clinical end points, including mortality. In the subsequent DETO2X-AMI trial to evaluate the effects of supplemental oxygen on hard clinical endpoints, Hofmann and colleagues (8) found that the routine use of supplemental oxygen in patients with suspected MI who did not have hypoxemia did not reduce or increase the 1-year all-cause mortality. When focusing on patients with ST-elevation MI, they found a similar result (9). Two meta-analyses of oxygen therapy in patients with acute MI reached the same conclusions, which were that oxygen therapy was not associated with important clinical outcomes in normoxemic patients $(10,11)$. Greater cardiac injury did not lead to more death in patients with acute MI. In general ICU patients, the limitations of the Oxygen-ICU trial were clear, and we are hoping to provide more evidence after conducting our registered Pulse Oxygen Saturation Directed Oxygen Therapy (POSDOT) trial. Along with POSDOT (NCT02999932), there are other ongoing large randomized controlled trials evaluating the efficacy of conservative oxygen therapy versus conventional oxygen therapy, for example, the ICU-ROX (ACTRN12615000957594), LOCO2 (NCT02713451), HOT-ICU (NCT03174002) and ICU-Conservative O2 (EUDRACT 2018-002525-35) trials (12).

For all patients included in our pilot study, the ICU mortality rate was $24.8 \%$, and the 28 -day mortality rate was $29.4 \%$, which coincided with our estimation and was reasonable for sample size calculation. As far as we know, the 28-day mortality rate in general ICU patients in China has not been reported before. The ICU mortality in our pilot study was higher than that in the study by Girardis and colleagues (11.6\% in the conservative group and $20.2 \%$ in the conventional group) (6), which could be explained by the severity of patients in the Chinese ICU compared with that in the European ICU (13).

Different $\mathrm{SpO}_{2}$ targets were used, especially in the low $\mathrm{SpO}_{2}$ group. Focusing on patients who were invasively ventilated, Panwar and colleagues $(5,14)$ set a target of $88-92 \%$ in the conservative oxygen group in comparison with $\geq 96 \%$ in the liberal group. Additionally, in mechanically ventilated patients, Suzuki and colleagues (15) performed a prospective before-after study, in which a $\mathrm{SpO}_{2}$ target of $90-92 \%$ in the conservative oxygen group was used. Although they did not evaluate the feasibility or efficacy of low $\mathrm{SpO}_{2}$ targeted oxygen therapy, Frat and colleagues (16) targeted $\mathrm{SpO}_{2} \geq 92 \%$ in spontaneously breathing patients with nonhypercapnic acute hypoxemic respiratory failure. Our $\mathrm{SpO}_{2}$ target in the low $\mathrm{SpO}_{2}$ group was relatively high, and $\mathrm{pO}_{2}$ was not targeted. Due to fear of hypoxia-induced injury, our ethics committee advised a $\mathrm{SpO}_{2}$ target of $90-95 \%$ instead of our planned target of $88-92 \%$ in the low $\mathrm{SpO}_{2}$ group. Our ethics committee also advised us not to change our routine care by performing more arterial blood gas analyses, since no compensation would 
be provided to the patients. Girardis and colleagues (6) had an even higher target in their conservative group $\left(\mathrm{SpO}_{2}\right.$ 94-98\%, $\mathrm{pO}_{2} 70-100 \mathrm{mmHg}$ ) and conventional control group $\left(\mathrm{SpO}_{2} 97-100 \%, \mathrm{pO}_{2}\right.$ up to $\left.150 \mathrm{mmHg}\right)$.

Although the $\mathrm{SpO}_{2}$ targets were able to be significantly differentiated between the two groups by only accommodating $\mathrm{FiO}_{2}$, most of the time, the mean $\mathrm{SpO}_{2}$ was higher than the predefined upper limit of the $\mathrm{SpO}_{2}$ target in the lower $\mathrm{SpO}_{2}$ group. The mean area under the curve for $\mathrm{SpO}_{2}$ was $93.4 \%$, but the target was $88-92 \%$ in the conservative oxygen group in Panwar and colleagues' study $(5,14)$. The median time-weighted average $\mathrm{SpO}_{2}$ in the conservative oxygen group was $95.5 \%$ in the study by Suzuki and colleagues (15), which was higher than the upper target limit of $92 \%$. In our study, the target was $90-95 \%$, but the time-weighted average $\mathrm{SpO}_{2}$ was $95.7 \%$ in the low $\mathrm{SpO}_{2}$ group. Further efforts are needed to strengthen compliance with the study protocol.

There were several important things worth noting while conducting multicenter trial. First, simple randomization may cause a difference in the number of patients between the two groups. A 1:1 ratio randomization stratified to study centers should be used in the upcoming multicenter randomized trial. Second, the time-weighted $\mathrm{pO}_{2}$ averages were not significantly different between the two groups, possibly because the time-weighted $\mathrm{SpO}_{2}$ average in the low $\mathrm{SpO}_{2}$ group was slightly higher than the upper limit of the $\mathrm{SpO}_{2}$ target or because $\mathrm{pO}_{2}$ was not the target in our study. Third, the low $\mathrm{SpO}_{2}$ group had more patients with respiratory failure than the high $\mathrm{SpO}_{2}$ group had $(\mathrm{P}=0.016)$. However, the percentage of patients receiving mechanical ventilation and APACHE II scores were not statistically different between the two groups $(\mathrm{P}=0.811, \mathrm{P}=0.302$, respectively). Fourth, during the screening process, more than half of the patients were excluded because of an expected ICU stay of less than 72 hours. This exclusion was reasonable because, in China, half of ICU patents, who are mainly postoperative patients, are discharged from the ICU within 24 hours (13).

\section{Conclusions}

$\mathrm{SpO}_{2}$ directed oxygen therapy in critical patients is feasible. Our pilot trial necessitates and rationalizes our largesample, multicenter trial.

\section{Acknowledgments}

We appreciate the help of all the personnel of our ICU in conducting this trial.

Funding: This work was supported by the Wu Jieping Medical Foundation (Grant number 320.6750.18011).

\section{Footnote}

Conflicts of Interest: The authors have no conflicts of interest to declare.

Ethical Statement: The authors are accountable for all aspects of the work in ensuring that questions related to the accuracy or integrity of any part of the work are appropriately investigated and resolved. The protocol had been previously approved by the ethics committee of Tongji Medical College, Huazhong University of Science and Technology, with which our hospital is affiliated (No. 2017-S024). Written informed consent was obtained from each patient's legal surrogate.

\section{References}

1. Stub D, Smith K, Bernard S, et al. Air versus oxygen in ST-segment-elevation myocardial infarction. Circulation 2015;131:2143-50.

2. Kilgannon JH, Jones AE, Shapiro NI, et al. Association between arterial hyperoxia following resuscitation from cardiac arrest and in-hospital mortality. JAMA 2010;303:2165-71.

3. Kilgannon JH, Jones AE, Parrillo JE, et al. Relationship between supranormal oxygen tension and outcome after resuscitation from cardiac arrest. Circulation 2011;123:2717-22.

4. Olasveengen TM, De Caen AR, Mancini ME, et al. 2017 International Consensus on Cardiopulmonary Resuscitation and Emergency Cardiovascular Care Science with Treatment Recommendations Summary. Circulation 2017;136:e424-40.

5. Panwar R, Hardie M, Bellomo R, et al. Conservative versus Liberal Oxygenation Targets For Mechanically Ventilated Patients: A Pilot Multicenter Randomized Controlled Trial. Am J Respir Crit Care Med 2016;193:43-51.

6. Girardis M, Busani S, Damiani E, et al. Effect of Conservative vs Conventional Oxygen Therapy on Mortality Among Patients in an Intensive Care Unit: The Oxygen-ICU Randomized Clinical Trial. JAMA 2016;316:1583-9.

7. Ferguson ND. Oxygen in the ICU: Too Much of a Good 
Thing? JAMA 2016;316:1553-4.

8. Hofmann R, James SK, Jernberg T, et al. Oxygen Therapy in Suspected Acute Myocardial Infarction. N Engl J Med 2017;377:1240-9.

9. Hofmann R, Witt N, Lagerquist B, et al. Oxygen therapy in ST-elevation myocardial infarction. Eur Heart J 2018;39:2730-9.

10. Sepehrvand N, James SK, Stub D. Effects of supplemental oxygen therapy in patients with suspected acute myocardial infarction: a meta-analysis of randomised clinical trials. Heart 2018;104:1691-8.

11. Li WF, Huang YQ, Feng YQ. Oxygen therapy for patients with acute myocardial infarction: a meta-analysis of randomized controlled clinical trials. Coron Artery Dis 2018;29:652-6.

Cite this article as: Yang X, Shang Y, Yuan S. Low versus high pulse oxygen saturation directed oxygen therapy in critically ill patients: a randomized controlled pilot study. J Thorac Dis 2019;11(10):4234-4240. doi: 10.21037/jtd.2019.09.66
12. Girardis M, Alhazzani W, Rasmussen BS. What's new in oxygen therapy? Intensive Care Med 2019;45:1009-11.

13. Du B, An Y, Kang Y, et al. Characteristics of critically ill patients in ICUs in mainland China. Crit Care Med 2013;41:84-92.

14. Mezidi M, Guérin C. Conservative versus liberal oxygenation targets for mechanically ventilated patients: A pilot multicentre randomized controlled trial. J Thorac Dis 2016;8:307-10.

15. Suzuki S, Eastwood GM, Glassford NJ, et al. Conservative oxygen therapy in mechanically ventilated patients: a pilot before-and-after trial. Crit Care Med 2014;42:1414-22.

16. Frat JP, Thille AW, Mercat A, et al. High-flow oxygen through nasal cannula in acute hypoxemic respiratory failure. N Engl J Med 2015;372:2185-96. 